\title{
Two-phase velocity measurement in a particle-laden jet
}

\author{
P. Liu ${ }^{1}$, K. M. Lam ${ }^{2, *}$
}

Submitted to: $\quad$ Journal of Hydro-Environment Research

(submitted: March 2011, revised: September 2011, final: January 2012)

Number of pages: $\quad 24$

Number of tables: 2

Number of figures: 15

${ }^{1}$ Graduate Student, ${ }^{2}$ Associate Professor,

Department of Civil Engineering, The University of Hong Kong, Pokfulam Road, Hong Kong

Corresponding author: Dr. K. M. Lam

Department of Civil Engineering, The University of Hong Kong, Pokfulam Road, Hong Kong, China

Tel: $\quad$ (852) 28591975

Fax: $\quad$ (852) 25595337

E-mail: kmlam@hku.hk

Keywords: Jets, Sediment, PIV, LIF 


\title{
Two-phase velocity measurement in a particle-laden jet
}

\author{
P. Liu, K. M. Lam
}

\begin{abstract}
A horizontally discharging jet laden with solid sediment particles is investigated experimentally. The submerged jet discharges water with an initially horizontal direction into stagnant water of the same density but the presence of sediment particles produces jet effluent having a combined density greater than that of the ambient water. A modified particle-imaging velocimetry (PIV) technique is applied to estimate the velocity fields of the solid particle phase and the jet fluid liquid phase. Phase separation is achieved optically between the scattered light signals from the particles and the laser-induced fluorescence signal from the jet fluid dozed with a fluorescent dye. It is found that initial sediment concentrations below $0.1 \%$ volume fraction do not cause significant changes to the global properties of the jet flow. In jets of higher initial sediment concentrations, settling of sand particles are observed to drag the jet to spread with a downward-bending mean trajectory. Intensive particle-flow interaction is also observed in jets of high sediment concentrations.
\end{abstract}

\section{Introduction}

Two-phase flows are found in many industrial and natural processes. The object of this paper is a particle-laden jet which can be observed in various areas of chemical engineering, environmental fluid mechanics and earth sciences. In environmental hydraulics, wastewater is discharged from outfalls in the form of a submerged jet into an ambient water body (Fischer et al., 1979). Residual solid particles are often present in the wastewater and the effluent is discharged as a particle-laden jet. In addition to the mixing and dilution of the liquid effluent phase, the fall out and settling of solid particles from the jet also give rise to many environmental 
problems, such as formation of sludge bank, consumption of dissolved oxygen in water body and even introduction of toxic materials to the food web of local ecosystem (Metcalf and Eddy, 1991).

A multiphase flow is more complex than a single-phase flow not only because of the presence of more constituents but also due to possible interaction among the different phases. In jets and plumes, particle-turbulence interaction leading to modifications of turbulent flow behavior has been observed (Gore and Crowe, 1989). Hence, studies of particle-laden flows not only provide information of the sediment-carrying jet flow, open channel flow or slurry pipe flow but also gives insights to the underlying mechanisms of particle-flow interaction which exist in a broad scope of multiphase flows. With limitations in theoretical treatments of phase interaction, researchers rely heavily on experimental investigations to study and derive empirical models for multiphase flow systems. The main challenge of any experimental study on multiphase flows is how to distinguish the flow signal of one phase while avoiding interference from those of the other phases. Once the signal of a particular phase is acquired, it can be measured and processed with various flow diagnostic techniques such as particle-image velocimetry (PIV) for the measurement of velocity vector field (Adrian, 2005) and laser induced fluorescence (LIF) for scalar concentration measurement (Crimaldi, 2008).

The non-intrusive laser-based flow diagnostic techniques of PIV and LIF have been applied to two-phase flow measurements. Previous works can be roughly categorized into two groups: (1) separation of phases by post-measurement imaging processing (e.g., Bryant et al., 2009), and (2) separation of phases by optical means during flow imaging (e.g., Jiang et al., 2005; Simiano et al., 2009). The first group of techniques recorded signals of the two phases on the same image while phase separation was achieved subsequently either by applying intensity difference or by image size differences (or by both). These post-measurement image processing algorithms usually requires a lot of computations. For the second group, images of the two phases will be separated by the optical setup before images are taken by a camera. The optical methods require more hardware but less computation. The separated images can be further processed to give velocity fields or scalar fields.

Our study targets on turbulent particle-laden jets discharging in a stagnant environment. Most previous investigations of this topic studied vertical round jets (e.g. Carey et al., 1988; Neves and Fernando 1995; Jiang et al., 2005), in which the discharging jet effluent and particle 
settling lie in the vertical direction and the mean flow is symmetrical. For a sediment-laden jet discharging in an initially horizontal direction, the effects of particle-flow interactions are more prominent because of particle falling out occurs more readily and the mean flow is always asymmetrical in the vertical direction. When modeling sediment jets or plumes, researchers often assume that sediment particles are fully dilute so that they do not have a significant effect on the main flow phase and have little particle-particle interaction (Bleninger et al., 2002; Lane-Serff and Moran, 2005; Li, 2006; Cuthbertson and Davies, 2008). Here, we would like to focus our investigation on the limiting concentration level above which effects of sediment particles on the jet flow cannot be neglected. Especially, we aim to investigate the effect of particle concentrations on the mean global behavior of the jet flow over a large region of the jet flow.

We list the particle characteristics in some previous investigations on particle-laden flows in Table 1. It is noted that the particle concentration in almost all studies in sediment-laden jets, plume and open channel flows was very low and not higher than $0.1 \%$ volume fraction $\left(c_{p}\right)$ while much higher particle concentrations were used to study slurry pipe flows. Municipal wastewater discharges typically have very low particle concentrations $\left(<1200 \mathrm{~g} / \mathrm{m}^{3}\right.$ ) (Metcalf and Eddy, 1991) but there may be situations such as slurry discharges or storm water discharges where particle concentrations and characteristics can vary widely.

Experimental studies of particle-laden jets have been based on point measurements (e.g., Barlow and Morrison, 1990; Calvo et al., 2009) and flow visualizations (e.g., Cardoso and Zarrebini 2001; Li, 2006), but only a limited number of studies presented global velocity measurement (e.g. Jiang et al., 2005; Sadr and Klewicki, 2005). In our experiments, the nonintrusive global velocity measurement technique of PIV is used to measure velocities of the two phases of a horizontally issued particle-laden jet. We separate flow images of the jet fluid phase and the sand particle phase with optical means and modified PIV techniques are applied to these images to estimate the velocity fields for each phase. The outcomes of the experiments are expected to reveal the main global features of the two phases and give some indications of particle-flow interaction in the scales of jet eddies. In addition to pure momentum jet discharge, we shall continue in the future to apply our technique to the investigation of buoyant discharge, which is of more interest in environmental hydraulics. 


\section{Experiment techniques}

\subsection{Experimental setup}

The experiments were conducted in a rectangular glass tank of length $1.8 \mathrm{~m}$, width $1.2 \mathrm{~m}$ and height $0.6 \mathrm{~m}$ (Fig. 1). The tank was filled with water to a depth of $0.54 \mathrm{~m}$ which was kept constant with an overflow device. A submerged round jet was formed by discharging water from a circular nozzle placed at mid-depth. The initial discharging direction was horizontal and the effluent water was supplied from a smaller overhead tank. Both ambient water and jet effluent are supplied from fresh water of density $\rho=998.2 \mathrm{~kg} / \mathrm{m}^{3}$ at $20^{\circ} \mathrm{C}$. Air conditioning was provided to the entire laboratory such that there were negligible changes in water temperature during the experiments. The jet nozzle had an exit diameter $D=7.5 \mathrm{~mm}$, which was preceded by a contraction of ratio 2.1:1 followed by short length of uniform cross section of diameter $D$. The flow rate of jet discharge and thus the jet exit velocity were controlled by the combination of a valve and a rotameter.

Natural sand grains were used as the sediment particles. The particles had a density at $\rho_{p}=$ $2.65 \times 10^{3} \mathrm{~kg} / \mathrm{m}^{3}$ and diameters ranging from 150 to $300 \mu \mathrm{m}$, with $d_{50}=225 \mu \mathrm{m}$. A vertical tube was placed $85 \mathrm{~mm}$ upstream of the jet nozzle to feed sediment particles into the supply pipe of jet fluid. The particle feed was metered by an hourglass placed near the end of the feed tube. Lee (1981) has showed that the feeding rate of sand particles was determined by the orifice diameter of the hourglass. For our experiments, we used a number of hourglasses of different orifice diameters to achieve the desirable particle concentration in the jet discharge.

We took a number of measures to promote good mixing of the particles with the jet fluid inside the jet nozzle so that the sediment-laden water would not become significantly stratified at the jet exit (Li, 2006). A steel mesh was placed in the effluent supply pipe before the particle feed location and generated turbulence to enhance fluid-particle mixing within the downstream portion of the supply pipe. Combined with the short residence time there, this prevented the particles from developing a stratified concentration before reaching the nozzle exit. The short parallel section, $0.7 \mathrm{D}$ in length, immediately upstream of the nozzle exit helped to make the particles exit with a horizontal flow direction. 
Distributions of particle concentration on the cross sections near the jet exit have been measured by Lee (2010) in a similar experimental setup (which was also used in Li, 2006). Measurements were made using both an optical technique based on particle counting with digital image processing and conventional suction tube samplings. The measured particle concentrations at cross sections near the jet exit showed a nearly circular distribution with the maximum concentration located very near $(x, y)=(0,0)$. It is thus deduced that the particle concentration at jet exit is very near uniform for the particle volume fractions used in $\mathrm{Li}$ (2006) and Lee (2010) which were less than or around $0.1 \%$. The range of volume fractions in the present study was beyond this value for which the condition of uniform particle concentration at jet exit had not been confirmed. Nevertheless, the main focus of the study is the global behavior of particle-laden jet due to particle loading and the effect of exit particle concentration may be of secondary importance.

Experiments were carried out at two values of jet exit velocity, $U_{j} \approx 0.45$ and $0.95 \mathrm{~m} / \mathrm{s}$, with the corresponding Reynolds numbers at $\operatorname{Re}=U_{j} D / v \approx 3750$ and 7950, where $v$ is the kinematic viscosity of water. For each jet exit velocity, sediment-laden jets with five values of particle concentration (in addition to the sediment-free pure jet) were tested and the volume fractions of particles are listed in Table 2. Table 2 also lists the relative density difference between the ambient fluid and the particle-laden effluent discharge, $\Delta \rho / \rho=\left(\rho_{\text {jet }}-\rho\right) / \rho$. The combined density of the effluent is computed from the particle fraction as: $\rho_{j e t}=C_{p} \rho_{p}+\left(1-C_{p}\right) \rho$, where $C_{p}$ is the volume fraction of sand particles. The densimetric

Froude number at jet exit, $F r=U_{j} / \sqrt{g D \Delta \rho / \rho}$, are also included in the table for later analysis purpose. It is noted that our particle concentration levels covered a range wider than most of the previous studies on sediment jets found in the literature (Table 1).

\subsection{Optical phase separation}

A double cavity Nd:YAG laser (Nano L50-100, Litron Lasers Ltd.) was employed as the light source. The maximum output per laser pulse was $50 \mathrm{~mJ}$ and the duration of the pulse was < $10 \mathrm{~ns}$. The dual laser pulses could be fired at a frequency up to $100 \mathrm{~Hz}$. The laser beam passed a set of optics including a cylindrical lens to generate a laser light sheet of uniform intensity. The 
light sheet was aligned to illuminate the vertical mid-longitudinal section of the jet flow field. Flow images on the illuminated plane were captured by a CCD camera of high quantum efficiency (HiSense MKII, Dantec Dynamics A/S). The camera had a resolution of $1344 \times 1024$ pixels and dynamic range of 12 bit grey scale for each pixel. In the experiments, the camera was operated at the double frame mode with a rate of 5 frame pairs per second. A gridded target was used to calibrate the field of interest. The field of view of the camera covered a region of $700 \times 530 \mathrm{~mm}^{2}$.

For the solid phase, the sediment particles scattered the laser light (green-colored at $532 \mathrm{~nm}$ wavelength) and registered themselves as bright spots. To distinguish the water phase, the jet effluent was dozed with the fluorescent dye Rhodamine 6G. When excited by the laser light, the dye emitted yellowish-colored fluorescence signals of longer wavelengths. A dye concentration at $200 \mu \mathrm{g} / \mathrm{L}$ was found sufficient to provide high enough fluorescence intensity to be recorded satisfactorily by the CCD camera. The separation of the two phases on the camera images was achieved by applying two optical filters, one being a narrow band pass filter which only passes the green light $(532 \mathrm{~nm})$ and the other a low pass filter, allowing only longer wavelengths (>560 $\mathrm{nm}$ ) to pass. In this set of our experiments, we only used one camera and the images for each phase were captured subsequently with the proper filter mounted in front of the camera. For measurement of each phase, 500 image pairs were captured. In each test run, image recording of length $100 \mathrm{~s}$ was taken about $1 \mathrm{~min}$ after initializing the jet flow. This was an optimization by considering the three aspects of full establishment of the jet flow, prevention of adverse particle stratification in the nozzle and minimization of dye accumulation inside the water tank.

Fig. 2 shows examples of flow images for each of the two phases. It can be observed that flow images for the two phases were successfully separated. In the image of the jet fluid phase (Fig. 2a), dye patches of different sizes which were carried by turbulent eddies outlined the jet flow region. It can also be observed that as the jet spreads downstream, it was gradually being bent downwards, apparently by the loading of the sand particles. In Fig. 2b, the sand particles revealed themselves as bright spots.

\subsection{PIV analysis of the two phases}


The solid phase of sediment was recorded as images of individual particles in our laser imaging (Fig. 2b). In the present particle-laden flow, a sand particle was not expected to move with a velocity very much different from those of its neighboring particles. Thus, we simply applied the standard digital PIV technique of interrogation windows and cross-correlation method (Willert and Gharib, 1991) to process the double-pulsed image pairs. The velocity vector so obtained in an interrogation window represented the averaged velocity of sediment particles in that small region. Although velocities of individual particles were not precisely known, this technique was found satisfactory in our particle-laden jets.

For the jet fluid phase, distinct dye patches could be observed in LIF images (Fig. 2a), especially in the mixing region of the jet. Thus, we regarded these small eddies or dye patches as PIV seeding particles and applied the cross-correlation based PIV algorithm on the double images. While the LIF signals provided sufficient contrast in the images to facilitate the crosscorrelation computation, the time interval between two pulses needed to be carefully adjusted so as to freeze the small eddies in each image while their displacements could still be detected from the double images. The time interval between the two laser pulses was set at $40 \mathrm{~ms}$ for both sets of measurements.

There have been previous attempts to compute velocity fields from image processing of visualization flow images ( $\mathrm{Su}$ and Dahm, 1996; Tokumaru and Dimotakis, 1995) and the technique is sometimes referred to as the scalar image velociometry (SIV). Law and Ho (2003) applied SIV based on a LIF setup to measure the velocity field of a jet flow and simultaneous measured the concentration field from LIF. For a fluid-particle two phase flow, the LIF technique has the special advantage of separating the signal of one phase from that of the mixture when laser is used as the source of illumination. In the present study, the modified PIV technique adopted for the fluid phase is similar to SIV in Law and Ho (2003). However, we did not attempt to measure the concentration field because the optical system was specifically set up for a relatively large field of interest and this incurred difficulties in accurate LIF calibration, especially with the presence of particles in the flow.

We tested our modified PIV analysis method on the fluid phase by taking measurement on a simple particle-free momentum round jet. The exit velocity was at $U_{j}=0.95 \mathrm{~m} / \mathrm{s}$. The PIV interrogation window was $32 \times 32$ pixels and this covered a physical area of $16.6 \times 16.6 \mathrm{~mm}^{2}$ which contained a number of jet eddies of dye patches. With $50 \%$ overlap among interrogation 
windows, $83 \times 63$ velocity vectors were obtained in a PIV snapshot, providing sufficient spatial resolutions for the study of jet global behavior. The PIV cross-correlation computation was carried out by the commercial software Dantec DynamicStudio. We used the adaptive crosscorrelation algorithm which incorporated multi-window and multi-pass procedures to increase the dynamical range of velocity determination (Scarano and Riethmuller, 1999). This was followed by velocity range validation to remove the obvious outlawed high velocity vectors.

Fig. 3a shows the instantaneous velocity field in a PIV snapshot obtained with our modified PIV method. While no valid vectors can be computed for the region outside the jet where there is no LIF signal (Fig. 2a), valid velocity vectors are also missing on various locations inside the jet. The most severe of these locations is the initial region of the jet, especially the potential core where the jet effluent is discharged uniformly and prior to any mixing with the ambient fluid; and hence no distinct eddy patches are available for PIV cross correlation analysis. Similarly, for those large dye-containing jet eddies, a valid PIV vector is not likely computed for an interrogation window located entirely inside an eddy. These missed vectors are usually found on interrogation windows near the jet centerline. The latter deficiency in using the dye patches as PIV seeding can be partially remedied when we compute the time-averaged mean velocity field from an ensemble of PIV snapshots. However, some of the PIV snapshots inevitably contain wrong vectors with very low velocities in points covered by a large dye patterns. This pulls down the mean velocities mainly at points near the jet centerline. Fig. $3 b$ shows the mean velocity field of our jet from averaging over 500 PIV snapshots. While the valid velocity vectors in the instantaneous PIV snapshot in Fig. 3a suffice to exhibit the turbulent actions of jet eddies and jet mixing, we can also observe the self-similar mean behavior of the evolving jet in the mean velocity field in Fig. 3b. Next, we shall analyze these self-similarities in order to validate the accuracy of our modified PIV technique.

It is well-accepted that $U_{c}(x)$ in the zone of established flow (ZEF) of a submerged round jet follows the $x^{-1}$ decay (Fischer et al., 1979; Lee and Chu, 2003). Fig. 4 plots the decay of mean velocity $U_{c}(x)$ along the jet centerline from our PIV results and the widely accepted relationship of:

$$
\frac{U_{c}}{U_{j}}=\frac{6.2}{x / D}
$$


This relation is obtained from theoretical analysis and extensive experiments of accurate point measurements. We can observe that our measured velocity data at the jet centerline agree with Eq. (1) only in the region at $x / D>60$. At more upstream locations, many large dye patches remain intact on the jet centerline and thus the mean velocities there are underestimated.

Fig. 5 presents the radial mean velocity profiles at some jet sections. The profiles are plotted in the usual non-dimensional form of $U(r) / U_{c}$ against $r / x$. Self-similarity of radial velocity profiles to the Gaussian distribution is expected in the ZEF (e.g., Fischer et al., 1979):

$$
U(x, r)=U_{c}(x) \exp \left[-\left(\frac{r}{b}\right)^{2}\right] \quad,
$$

where $b$ is the jet half width. At $r=b$, the mean concentration equals $1 /$ e times the concentration $U_{c}$ at the jet centerline. The jet half width increases linearly with $x$ as $b=\beta x$, where $\beta$ is the spreading rate. We adopt the value of $\beta=0.114$ as determined empirically in Lee and Chu (2003) to plot the similarity profiles in Fig. 5. Due to the errors in the measured centerline velocities at some upstream stations, the values of $U_{c}$ used for the normalization of $U(r)$ are obtained from Eq. (1) instead. This is expected to provide a truer validation of the measured mean velocities from our PIV method at locations away from the jet centerline. At the sections of $x / D=31.6$ and 37.1, the measured mean velocities near the jet centerline are below the Gaussian profile as expected but at other radial locations the measured velocities match Eq. (2) satisfactorily. The diffusion of dye patterns at those locations makes PIV computation reliable (Fig. 2a). At the more downstream sections of $x / D>48.2$, the technique works well and the measured data follows Eq. (2) nicely. It is noted that outside the jet flow region, at $|r| x \mid>0.3$, the mean velocity profiles fail to approach zero. This non-zero velocity is probably due to the increasing probability of absence of LIF signal along the radial direction and it is also observed by Law and Ho (2003).

We continue to investigate the turbulent stresses measured by our PIV technique. Fig. 6 shows the radial profiles of the three turbulent stresses, together with the hot-wire measurement data of Wygnanski and Fiedler (1969). In general, self-similarity is observed on the radial profiles of the three stresses in regions between $x / D=31.6$ and 79.1 but the levels are lower than the hot-wire data. Webster et al. (2001) reported PIV measurement of these turbulent stresses. For the axial normal stress $\overline{u^{\prime} u^{\prime}}$, those PIV data were also below the hot-wire data in Fig. 6 with 
peak values in the range of $0.24-0.3$. Our present data are even lower but they exhibit the same self-similar shape as the previous two studies (Fig. 6a). We believe that our PIV method based on the dye patches is poor in detecting velocity fluctuations of small scales, thus resulting in significant underestimation of turbulent stresses. This problem is worse for the radial normal stress $\overline{v^{\prime} v^{\prime}}$ in Fig. $6 \mathrm{~b}$ in which our values are only about $50 \%$ of the hot-wire data. This may be because large eddies move mainly in the axial direction and the time interval between an image pair has been chosen by this consideration, leading to more severe deficiency in detecting radial velocity fluctuations. For the same reason, the turbulent shear stress $\overline{u^{\prime} v^{\prime}}$ is also severely underestimated (Fig. 6c) but their self-similar profiles agree well with that of the hot-wire data with the peak shear stress located at $r / x= \pm 0.1$. These correspond to the locations where the velocity gradient is the greatest.

The test measurement of a simple jet in Figs. 3-6 show that the present modified PIV method applied to LIF dye patches fails to get accurate velocity measurement at locations outside the jet region, inside the potential core and at regions where there are insufficient diffusion and breakdown of dye patches. However, satisfactory estimation of mean velocities can be made after ensemble time averaging and at regions more downstream of the jet exit and in the mixing region of the jet. More importantly, the measurement method succeeds in reproducing the radial profiles of mean velocities and turbulent stresses. Thus, we can locate the jet trajectories and jet widths with confidence from the measurements. These are essentially the information we aim to obtain in later sections for the jet fluid phase of a particle-laden jet.

As an alternative, fluorescent seeding particles are ideal to mark the jet fluid phase for PIV analysis (Pedocchi et al., 2008) but in our application targeting at a large jet flow area, their small sizes cannot produce sufficiently strong fluorescence signal on the camera. Another drawback is the high cost of the fluorescent particles.

\section{Results and discussion}

\subsection{Jet flow field}


For the sediment-laden jets in Table 2, we carried out two-phase PIV measurements where the modified PIV method was used on the jet fluid phase with LIF signal. Results of mean velocity fields obtained for the jet fluid phase of four jets at $U_{j}=0.95 \mathrm{~m} / \mathrm{s}$ but with different particle concentrations are shown in Fig. 7. The flow field for $C_{p}=0.07 \%$ is almost identical to that of the pure jet (Fig. 3b) and this not shown. We can observe that when the particle concentration increases, especially up to $0.12 \%$ in volume fraction, the jet is obviously bent downwards. At the same time, the lower half of the jet section is stretched, probably due to the falling sediment particles and the radial velocity profiles become more asymmetrical with the increase of particle load. These two effects of the particles on the mean jet behavior can also be observed from the corresponding LIF visualizations. Fig. 8 shows these mean LIF images obtained from simple ensemble averaging of the instantaneous LIF images such as the one in Fig. 2a. The LIF images are used in our modified PIV analysis but they are not suitable for evaluation of jet effluent concentration. This is because of the difficulty in calibration of LIF levels against jet effluent concentration in the situation of our large measurement area and in the presence of sand particles. However, since the laser sheet was shone along a roughly horizontal direction from the upstream side of the jet, the laser intensity remained nearly uniform along the vertical direction at an axial location. Thus, we can reliably determine the jet trajectory and jet widths from the mean LIF level distribution in a similar manner as velocity measurement by our modified PIV method.

We find the jet centerline trajectories by locating the points of maximum mean velocity and maximum mean LIF levels in their vertical profiles at successive jet sections. The results are shown in Fig. 9 and it is evident that the trajectories from the two profiles agree with each other in terms of bending magnitude for the same particle-laden jet. This provides an indirect confirmation of the success of our phase separation method and PIV treatment of LIF images based on the movement of dye patches. Fig. 9a,b shows that the trajectory of the jet with particle concentration at $C_{p}=0.07 \%$ is almost identical as that of the sediment-free simple jet. This is consistent with the findings on the literature that sediment particles at concentration of $0.1 \%$ or below do not have significant effect on the jet mean behavior (Cuthbertson and Davies, 2008). For jets with particle concentrations higher than $0.12 \%$, differences in jet trajectories from the simple jet case can no longer be neglected. 
It is interesting to check whether the effect of particle loads in drawing the jet downwards can be represented by the negative buoyancy effect from the combined density of particle-laden jet effluent. We compute the trajectory of negatively buoyant jets of the same combined densities and corresponding Froude numbers as listed in Table 2 with the Lagrangian integral model JETLAG (Lee and Cheung, 1990; Lee and Chu, 2003). For clarity, only the jet trajectory computed for a negatively buoyant single-phase jet with equivalent bulk effluent density as the sediment jet with $C_{p}=0.47 \%$ is included in Fig. 9a. It is evident that the equivalent negatively buoyant jet has a much greater downward bending trajectory than the particle-laden jet.

In a related study, we also attempted some computational fluid dynamics (CFD) simulations of horizontally issued particle-laden jets using a simple inter-phase interaction model by way of the drag law to account for the phase interaction. The CFD results (to be reported in a future paper) are able to better match the experimental data of jet centerline trajectories. The result for $C_{p}=0.47 \%$ is included in Fig. 9a for illustration. It is thus clear that the effect of particle loading should be modelled by the physical interaction between the fluid phase and the solid particles rather than using the bulk density difference.

Fig. 10 shows the decay of maximum mean velocity at successive jet sections along the downstream direction in jets with different particle concentrations. It can be observed that the fluid velocities on the jet trajectories are only slightly reduced by the presence of sediment particles. Fig. 11 shows the vertical profiles of mean velocity component $u$ at $x / D=60$ and 80 of the sediment jets. We can evidently observe the increasing downward shifting of the profiles with increases in particle loading. As already found in Fig. 10, the maximum velocities in the profiles are slightly reduced from the simple jet case. Fig. 11 also shows that on the lower side of the jet, the width of the sediment-laden jet is wider than that of a simple jet.

\subsection{Velocity field of particles}

In our two-phase PIV measurements of particle-laden jets, the average velocity of sediment particles inside an interrogation window is found from the PIV analysis of particle images scattering laser light. The mean particle velocity field is obtained from the ensemble of 500 PIV snapshots and the results of four jets are shown are shown in Fig. 12. In regions above the jet, some spurious vectors can be observed. These are mainly caused by the lack of sediment 
particles there for PIV analysis. Nevertheless, these wrong vectors are in regions of less interest and can be omitted in subsequent analyses. The jet flow fields of these jets have been presented in Fig. 7. Comparing the two velocity fields, we can observe that in upstream part of our measurement region, say at $x / D<50$, the location of maximum particle velocity across a vertical jet section is near to that of maximum fluid velocity but at farther downstream locations, the sectional maximum particle velocities seem to occur at a lower location. Particles are observed to fall from the lower edge of the jet continuously and starting right from the jet exit. It appears that for a jet carrying a higher initial particle concentration, fewer portions of the particles can be transported downstream as compared with one with a lower initial particle concentration. We cannot observe a self-similar behavior of particle motion at successive axial stations, especially in the lower half of the jet. Instead, we observe that particles after falling out from the jet tend to settle eventually with a vertical velocity of constant magnitude in the region beneath the jet and bounded by the bottom of the water tank.

We estimate the settling velocity of the particles after they have fallen out of the jet for some distances by averaging the particle velocity vectors inside the region enclosed by dashed lines in each plot of Fig. 12. The values are shown for the four jets. The selected region is far below the jet region and the average velocity is expected to approach the settling velocity of particles which are free from influence of the jet flow. The expected settling velocity of a single particle or widely separated particles in a stagnant flow is given by Stokes' law as: $w_{s}=\frac{2}{9} \frac{(s-1)}{v} g d^{2}$, where $s$ is the relative density of the particles. When we use $d_{50}$ of our sand particles as the particle diameter $d$, this equation gives $w_{s}=0.182 \mathrm{~m} / \mathrm{s}$, which is several times higher than the falling velocities in Fig. 12. This disagreement is expected because Stokes' law assumes low-velocity laminar flow around a single spherical particle. Soulsby (1997) proposed the following expression for particle settling velocity in still water for natural sand grains by considering a combination of skin friction and bluff-body drag:

$$
w_{s}=\frac{v}{d}\left[\left(10.36^{2}+1.049 D_{*}^{3}\right)^{1 / 2}-10.36\right]
$$

The dimensionless diameter, $D_{*}$ is defined as: $D_{*}=\left[\frac{g(s-1)}{v^{2}}\right]^{1 / 3} d$. The settling velocity from Eq. (3) is $w_{s}=0.031 \mathrm{~m} / \mathrm{s}$. This is close to the falling velocities, $U_{s}$, from our PIV measurement of 
sand particle phase for the two jets with lower initial particle concentrations (Fig. 12a-b). The lower values of our falling velocities may be caused by the interaction of flows around adjacent sand particles during settling. Some evidence can be observed in Fig. $2 b$ in which the settling particles in the region of interest show patterns of "fingers" as reported by Cardoso and Zarrebini (2001) as results of particle-eddy interaction in a jet flow. On the other hand, values of $U_{s}$ significantly higher than $0.031 \mathrm{~m} / \mathrm{s}$ are found in the two jets with higher initial particle concentrations (Fig. 12c-d). We suspect that this is caused by some aggregation of particles upon settling. There may also be a role played by turbulence-particle interaction which will be discussed in the next subsection.

Fig. 13 shows the vertical profiles of velocity magnitude of the two phases at $x / D=65$ for two sediment-laden jet with similar initial particle concentrations but different jet exit velocities. We can observe that in the jet with the higher $U_{j}=0.95 \mathrm{~m} / \mathrm{s}$ the profiles are peaked at an upper location than that of the jet with the lower exit velocity. The result is expected and may be used to validate length scale analyses or numerical models in the study of sediment-laden jets.

\subsection{Turbulence-particle interaction}

From the ensemble of 500 PIV snapshots of jet fluid velocity fields, we compute the Reynolds turbulent stresses. Fig. 14 shows the vertical profile of axial stresses at the section $x / D$ $=40$ for a simple jet free of particles and jets laden with particles of different initial concentrations. It is observed that particle loading leads to increase in turbulence intensity of the jet fluid flow phase. This result is also supported by flow visualizations in Fig. 15 shown for the jet with very high $C_{p}=0.77 \%$. Very different from the jet with of lower particle concentration in Fig. 2, we can observe large organized clouds of particles in Fig. 15b. Although we did not obtain velocities of the two phases simultaneously, we believe that there exist strong relation and interaction between the clouds structures of particles and the large-scale turbulent eddies of the jet (Fig. 15a). The result appears to disagree with Gore and Crowe (1989) who stated that larger particles increase the turbulence intensity of the flow while small particles decrease the turbulence intensity. We suspect that with a higher initial particle concentration in the sediment jet, more particles can interact with the coherent structures of the jet and they joint action to extract energy from the mean flow to turbulence energy. In the next stage of this study, we plan 
to carry out simultaneous two-phase measurements to further investigate this important phenomenon which may largely affect the particle falling out mechanism.

\section{Conclusions}

Sediment-laden jets with different initial particle concentrations are studied with laser imaging techniques. The solid phase and the fluid phase are separated optically in the imaging by the difference in wavelengths between the laser light scattered by the sediment particles and the fluorescence light emitted by dye-containing jet fluid. Velocity fields of the particle and jet fluid phases are estimated with the PIV technique based on double-image cross-correlation. For the particle phase, the technique measures the average velocity of particles inside small interrogation windows while for the jet fluid phase, dye patches carried by eddy eddies are taken to be a form of PIV seeding. The modified PIV method for the fluid phase velocities is tested and validated through measurements on a simple momentum round jet free of particles of which the global flow behavior is well known. The result shows that the technique provides reliable mean velocity measurements in the mixing region of the jet where there exist sufficiently diffused dye patterns broken up by jet eddies. The technique is able to obtain the correct distribution of radial profiles of mean velocity and, in a less accurate degree, turbulent stresses. Thus, reliable estimations of jet trajectories and jet widths can be made.

The modified PIV technique is applied for two-phase velocity measurements of a number of particle-laden jets of varying initial particle concentrations. It is found that sediment particles of initial concentration not higher than $0.1 \%$ in volume fraction do not lead to significant modification of global properties of the jet. This finding is consistent with those of previous reported studies. A jet laden with a higher particle concentration is found to have its jet centerline trajectory bent downwards by the sediment load. The degree of this bending from the particle load is smaller than that of an equivalent negative buoyant jet with effluent of the same combined density of the fluid-particle discharge. The velocity width on the lower part of the jet is also increased by the sediment load. At a high particle concentration, say, $0.77 \%$ in volume fraction, intensive particle-flow interaction is observed and will be investigated in the future. 


\section{Acknowledgement}

This investigation is supported by a research grant (HKU 7194/08E) awarded by the Research Grants Council of Hong Kong. 


\section{References}

Adrian, R.J., 2005. Twenty years of particle Image velocimetry. Exp. Fluids 39, 159-169.

Barlow, R.S., Morrison, C.Q., 1990. Two-phase velocity measurement in dense particle-laden jets. Exp. Fluids 9, 93-104.

Bleninger, T., Carmer, C.V., Jirka, G., Neves, M., 2002. Sedimentation from low concentration particle-laden jets. Proc., 2nd Int. Conf. Marine Waste Water discharges, Istanbul.

Bryant, D.B., Seol, D.G., Socolofsky, S.A., 2009. Quantification of turbulence properties in bubble plumes using vortex identification metods. Phys. Fluids 21 (075101), 1-10.

Calvo, E., Garcia, J.A., Garcia, I., Aisa, L.A., 2009. Errors analysis in the evaluation of particle concentration by PDA on a turbulent two-phase jet: application for cross section and transit time methods. Exp. Fluids 47, 489-508.

Cardoso, S.S.S., Zarrebini, M., 2001. Convection driven by particle settling surrounding a turbulent plume. Chem. Eng. Sci. 56, 3365-3375.

Carey, S.N., Sigurdsson, H., Sparks, R.S.J., 1988. Experimental studies of particle-laden plumes. J. Geophysical Res. 15, 314-328.

Crimaldi, J.P., 2008. Planar laser induced fluorescence in aqueous flows. Exp. Fluids 44, 851863.

Cuthbertson, A.J.S., Davies, P.A., 2008. Deposition from particle-laden, round, turbulent, horizontal, buoyant jets in stationary and coflowing receiving fluids. J. Hydraul. Eng. 134(4), 390-420.

Fischer, H.B., List, E.J., Koh, R.C.Y., Imberger, J., Brooks, N.H., 1979. Mixing in inland and coastal waters. Academic Press, New York, N.Y.

Gore, R.A., Crowe, C. T., 1989. Effect of particle size on modulating turbulent intensity. Int. J. Multiphase Flow 15(2), 279-285.

Jiang, J., Law, A.W.K., Cheng, N.S., 2005. Two-phase analysis of vertical sediment-laden jets. J. Eng. Mech. 131, 308-318. 
Lane-Serff, G.F., Moran, T.J., 2005. Sedimentation from buoyant jets. J. Hydraul. Eng. 131(3), 166-174.

Law, A.W.-K., Ho, W.F., 2003. Simultaneous velocity and concentration measurements with scalar image velocimetry and PLIF. Proc., 7th Asian Symposium on Visualization, Singapore.

Lee, J.H.W., 1981. An hourglass experiment. Int. J. Mech. Eng. Educ. 9(1), 39-46.

Lee. J.H.W., Cheung, V., 1990. Generalized Lagrangian model for buoyant jets in a current. J. Environ. Eng. ASCE 166(6), 1085-1106.

Lee, J.H.W., Chu, V.H., 2003. Turbulent jets and plumes - a Lagrangian approach. Kluwer Academic Publishers, Boston.

Lee, W.Y., 2010. Mixing of horizontal sediment laden jets. PhD Thesis, The University of Hong Kong, China.

Li, A.C.Y., 2006. Theoretical modeling and experimental studies of particle-laden plumes from wastewater discharges. M. Phil. Thesis, The University of Hong Kong, Hong Kong, China.

Lyn, D.A., 1992. Turbulence characteristics of sediment-laden flows in open channels. J. Hydraulic Eng. 118, 971-988.

Metcalf and Eddy Inc., 1991. Wastewater engineering: treatment, disposal, reuse. McGraw-Hill Inc., Singapore.

Martin, D., Nokes, R., 1988. Crystal settling in a vigorously convecting magma chamber. Nature $332,534-536$

Matousek, V., 2002. Pressure drops and flow patterns in sand-mixture pipes. Exp. Thermal and Fluid Sci. 26, 693-702.

Neves, M.J., Fernando, H.J., 1995. Sedimentation of particles from jets discharged by ocean outfalls: a theoretical and laboratory study. Wat. Sci. Tech. 32, 133-139.

Noguchi, K., Nezu, I., 2009. Particle-turbulence interaction and local particle concentration in sediment-laden open channel flows. J. Hydro-environ. Res. 3, 54-68. 
Pedocchi, F., Martin, J.E., Garcia, M.H., 2008. Inexpensive fluorescent particles for large-scale experiments using particle image velocity. Exp. Fluids 45, 183-186.

Sadr, R., Klewicki, J.C., 2005. Flow field characteristics in the near field region of particle-laden coaxial jets. Exp. Fluids 39, 885-894.

Scarano, F., Riethmuller, M.L., 1999. Iterative multigrid approach in PIV image processing with discrete window offset. Exp. Fluids 26, 513-523.

Simiano, M., Lakehal, D., Lance, M., Yadigaroglu, G., 2009. Turbulent transport mechanisms in oscillating bubble plumes. J. Fluid Mech. 633, 191-231.

Soulsby, R., 1997. Dynamics of marine sands. Thomas Telford Ltd., London, UK.

Su, L.K., Dahm, W.J.A., 1996. Scalar image velocimetry measurements of the velocity gradient tensor field in turbulent flows. II. Experimental results. Phys. Fluids 8, 1883-1906.

Tokumaru, P.T., Dimotakis, P.E., 1995. Image correlation velocimetry. Exp. Fluids 19, 1-15.

Webster, D.R., Roberts, P.J.W., Ra'ad, L., 2001. Simultaneous DPTV/PLIF measurements of a turbulent jet. Exp. Fluids 30, 65-72.

Willert, C.E., Gharib, M., 1991. Digital particle image velocimetry. Exp. Fluids 10, 181-193.

Wilson, K.C., Pugh, F.J., 1988. Dispersive force basis for concentration profiles. J. Hydraulic Eng. $114,806-810$.

Wynanski, I., Fiedler, H., 1969. Some measurements in the self-preserving jet. J. Fluid Mech. 38, 577-612. 


\section{Tables}

Table 1. Previous studies of particle-laden flow.

\begin{tabular}{|c|c|c|c|c|}
\hline \multirow[b]{2}{*}{ Literature } & \multicolumn{3}{|c|}{ Particle Properties } & \multirow[b]{2}{*}{ Application } \\
\hline & Diameter $d_{p}(\mathrm{~mm})$ & $\begin{array}{l}\text { Density } \rho_{p} \\
\left(\mathrm{~g} / \mathrm{cm}^{3}\right)\end{array}$ & $\begin{array}{l}\text { Vol. fraction } \\
\qquad C_{p}(\%)\end{array}$ & \\
\hline $\begin{array}{l}\text { Wilson and Pugh } \\
\text { (1988) }\end{array}$ & $0.1,0.2,0.5,1.0$ & 2.65 & $0 \sim 60$ & Slurry pipe flow \\
\hline Matousek (2002) & $0.12,0.37,1.85$ & 2.65 & $12, . ., 26$ & Slurry pipe flow \\
\hline Lyn (1992) & $0.15,0.19,0.24$ & 2.65 & $<0.19$ & Open channel flow \\
\hline $\begin{array}{c}\text { Noguchi and Nezu } \\
\text { (2009) }\end{array}$ & $\begin{array}{c}0.25,0.37,0.5 \\
1.0\end{array}$ & $1.2, . ., 1.5$ & $0.03 \sim 0.29$ & Open channel flow \\
\hline $\begin{array}{l}\text { Martin and Nokes } \\
\qquad(1988)\end{array}$ & $\begin{array}{l}0.21 \sim 0.31 \\
0.31 \sim 0.42 \\
0.42 \sim 0.50\end{array}$ & 1.033 & 0.3 & Thermal tank \\
\hline $\begin{array}{l}\text { Carey et al. } \\
\text { (1988)* }\end{array}$ & $0.007 \sim 0.120$ & 3.21 & $<1.87$ & Vertical Sediment plume \\
\hline $\begin{array}{c}\text { Neves and } \\
\text { Fernando (1995) }\end{array}$ & $\begin{array}{c}0.530,0.799 \\
0.868\end{array}$ & $\begin{array}{l}1.0445 \\
1.0251\end{array}$ & $\begin{array}{l}0.0045 \sim 0.2 \\
3\end{array}$ & Vertical Sediment jet \\
\hline Jiang et al. (2005) & 0.075 & 1.51 & 0.19 & Vertical Sediment jet \\
\hline $\begin{array}{l}\text { Lane-Serff and } \\
\text { Moran (2005) }\end{array}$ & $\begin{array}{c}0.075 \sim 150, \\
0.150 \sim 0.300\end{array}$ & 2.65 & $<0.38$ & Angled Sediment jet \\
\hline $\begin{array}{l}\text { Bleninger et al. } \\
\qquad(2002)\end{array}$ & $0.45 \sim 0.50$ & 1.022 & $0.02 \sim 0.47$ & Horizontal Sediment jet \\
\hline Li (2006) & $\begin{array}{l}0.063 \sim 0.150 \\
0.150 \sim 0.212\end{array}$ & 2.65 & $0.15 \sim 0.17$ & Horizontal Sediment jet \\
\hline $\begin{array}{l}\text { Cuthbertson and } \\
\text { Davies (2008) }\end{array}$ & $\begin{array}{c}0.500 \sim 0.600, \\
0.630 \sim 0.850\end{array}$ & $1.15, \quad, 1.50$ & $\sim 0.1$ & Horizontal Sediment jet \\
\hline
\end{tabular}

* Ambient salt water density: $1.021 \mathrm{~g} / \mathrm{cm}^{3}$. 
Table 2. Experimental parameters.

\begin{tabular}{|c|c|c|c|c|c|}
\hline$U_{j}=0.95 \mathrm{~m} / \mathrm{s}$ & & & & & \\
\hline volume fraction $(\%)$ & 0.07 & 0.12 & 0.25 & 0.47 & 0.77 \\
\hline$\Delta \rho / \rho(\%)$ & 0.12 & 0.20 & 0.41 & 0.78 & 1.27 \\
\hline $\mathrm{Fr}$ & 103.1 & 78.7 & 54.5 & 39.8 & 31.1 \\
\hline \multicolumn{6}{|l|}{$U_{j}=0.45 \mathrm{~m} / \mathrm{s}$} \\
\hline volume fraction (\%) & 0.15 & 0.25 & 0.52 & 0.98 & 1.63 \\
\hline$\Delta \rho / \rho(\%)$ & 0.25 & 0.41 & 0.86 & 1.62 & 2.69 \\
\hline$F r$ & 33.3 & 25.8 & 17.9 & 13.0 & 10.1 \\
\hline
\end{tabular}




\section{Figure Captions}

Fig. 1. Experimental setup.

Fig. 2. Optical separation of jet fluid phase and solid particle phase. Sediment jet with $C_{p}=$ $0.25 \%$.

Fig. 3. Flow field of a particle-free momentum jet: (a) instantaneous velocity field; (b) mean velocity field.

Fig. 4. Centerline velocity decay in a particle-free momentum jet.

Fig. 5. Radial velocity profiles in a particle-free momentum jet.

Fig. 6. Turbulent Reynolds stresses in a particle-free momentum jet. Symbols same as Fig. 5. Lines are data of Wygnanski and Fiedler (1969).

Fig. 7. Mean velocity field of jet fluid flow in sediment jets.

Fig. 8. Mean LIF visualization of jet fluid flow.

Fig. 9. Centerline trajectories of sediment jets: (a) from mean velocity field; (b) from mean LIF visualization. NBJ: JETLAG prediction of equivalent negatively buoyant single-phase jet; CFD: CFD result using drag forces between phases.

Fig. 10. Centerline velocity decay in sediment jets.

Fig. 11. Vertical profiles of mean axial fluid velocity: (a) $x / D=60$; (b) $x / D=80$.

Fig. 12. Average velocity field of sediment particles. 
Fig. 13. Comparison between vertical profiles of mean fluid velocity and particle velocity at $x / D$ $=65$. Open symbols: $U_{j}=U_{1}=0.95 \mathrm{~m} / \mathrm{s}$ and $C_{p}=0.47 \%$; solid symbols: $U_{j}=U_{2}=0.45$ $\mathrm{m} / \mathrm{s}$ and $C_{p}=0.52 \%$.

Fig. 14. Turbulence intensity affected by particle loading. $x / D=40$.

Fig. 15. Intense interaction shown from visualization. $C p=0.77 \%$. 


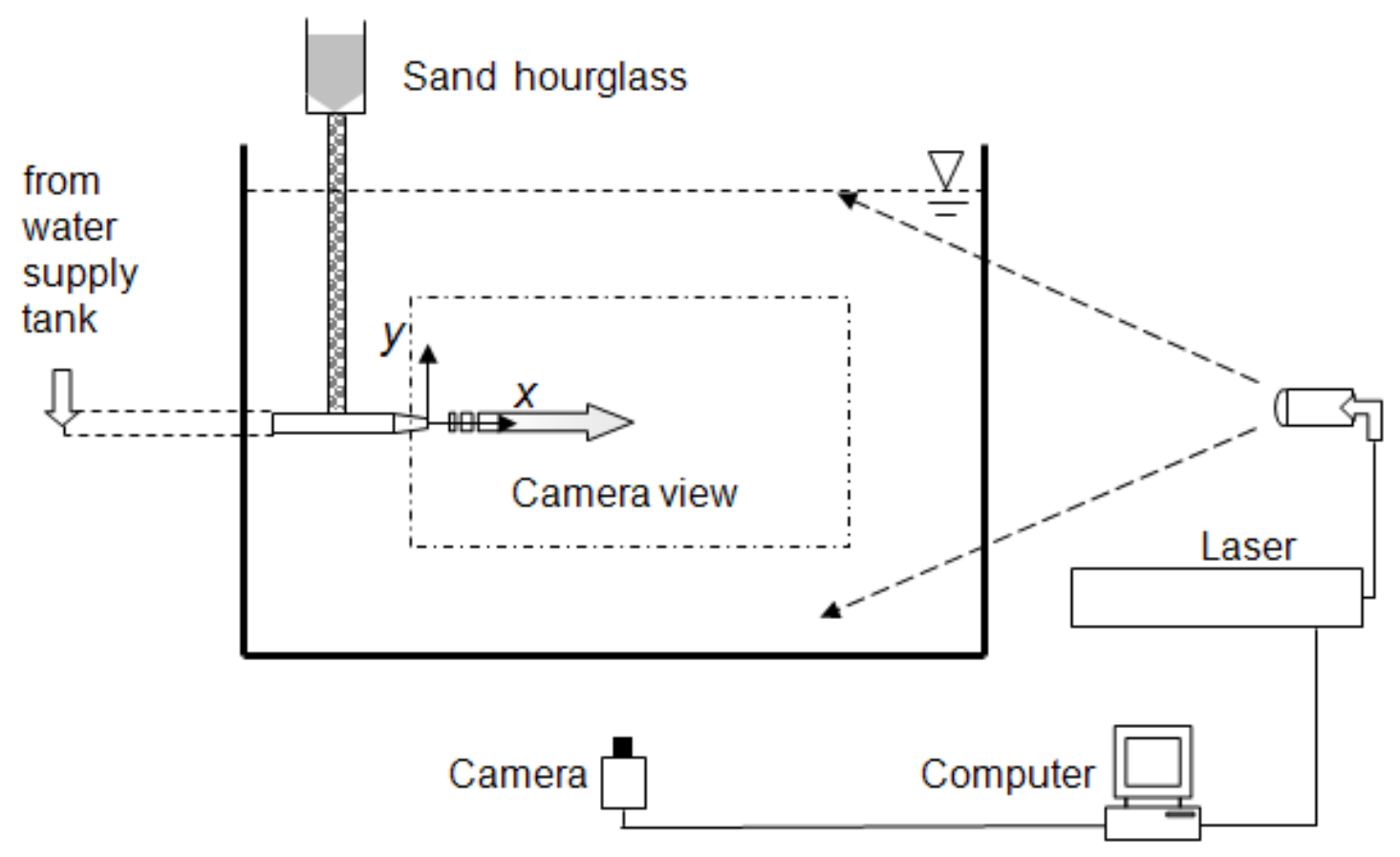

Fig. 1. Experimental setup. 

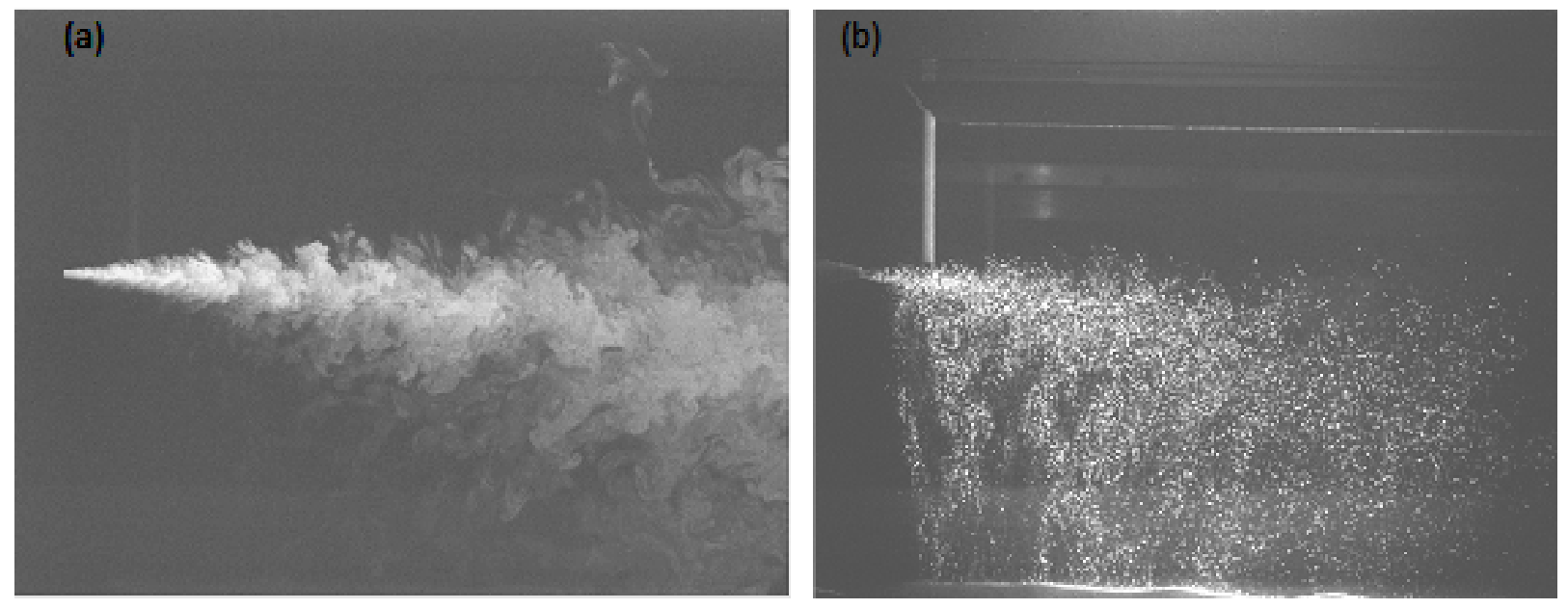

Fig. 2. Optical separation of jet fluid phase and solid particle phase. Sediment jet with $C_{p}=0.25 \%$. 

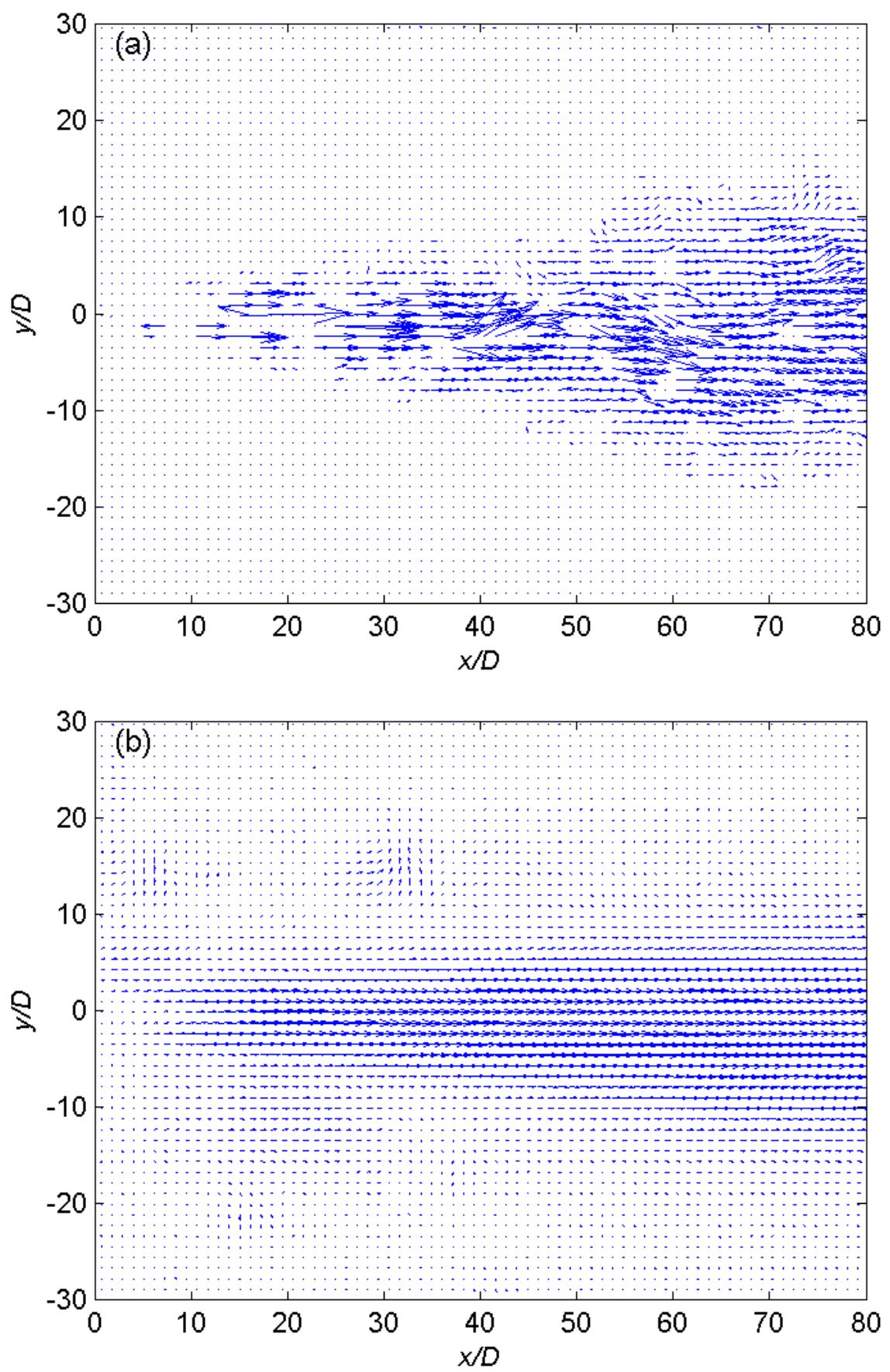

Fig. 3. Flow field of a particle-free momentum jet. (a) instantaneous velocity field; (b) mean velocity field. 


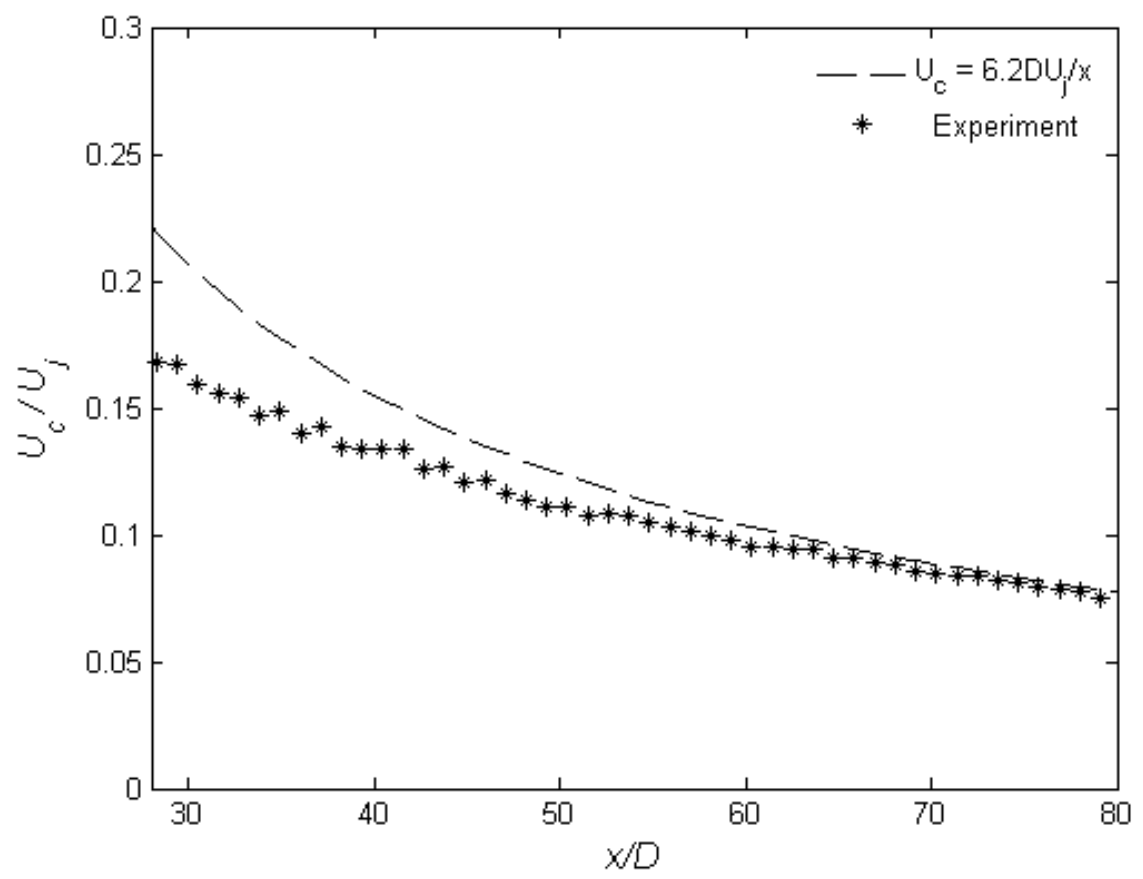

Fig. 4. Centerline velocity decay in a particle-free momentum jet. 


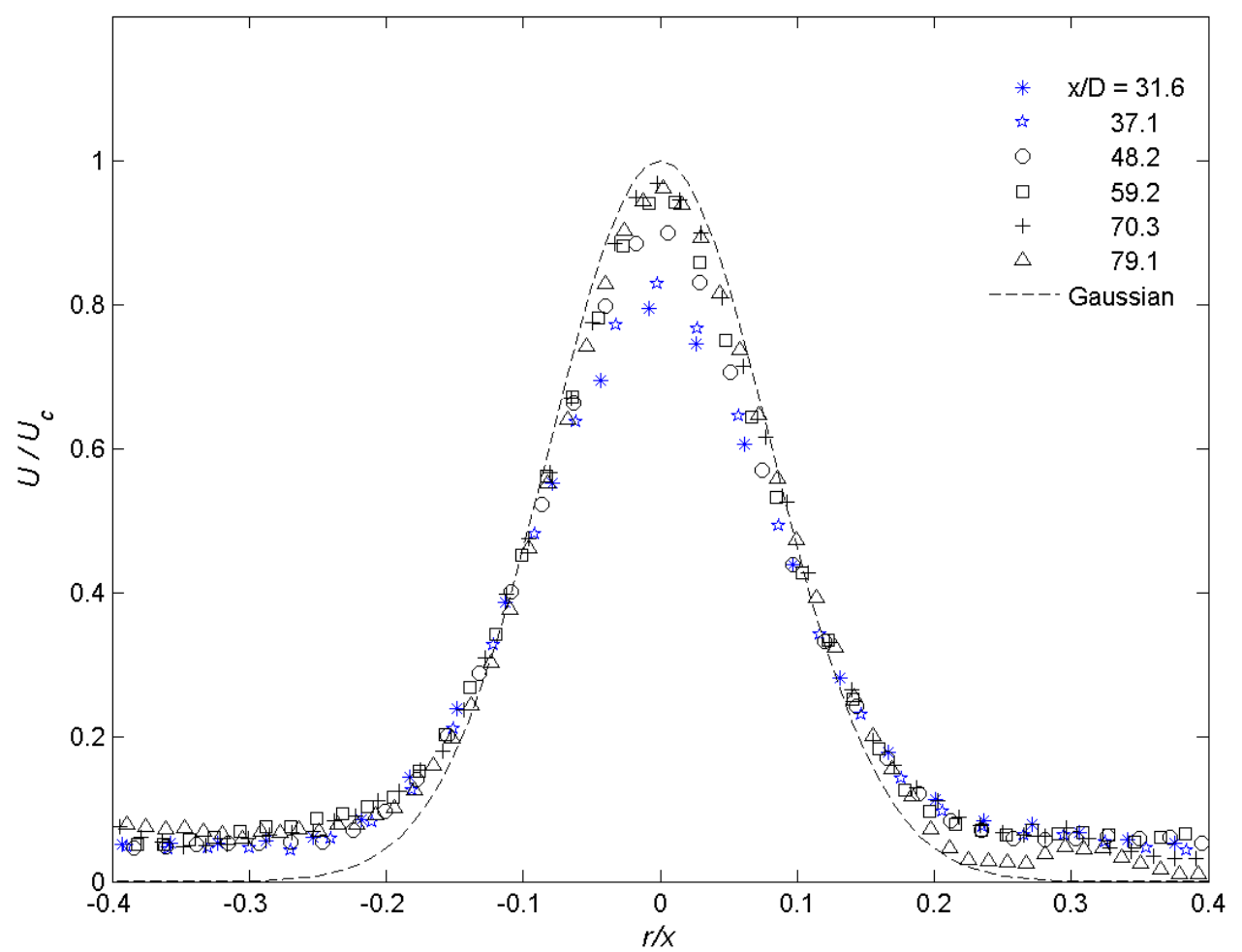

Fig. 5. Radial velocity profiles in a particle-free momentum jet. 

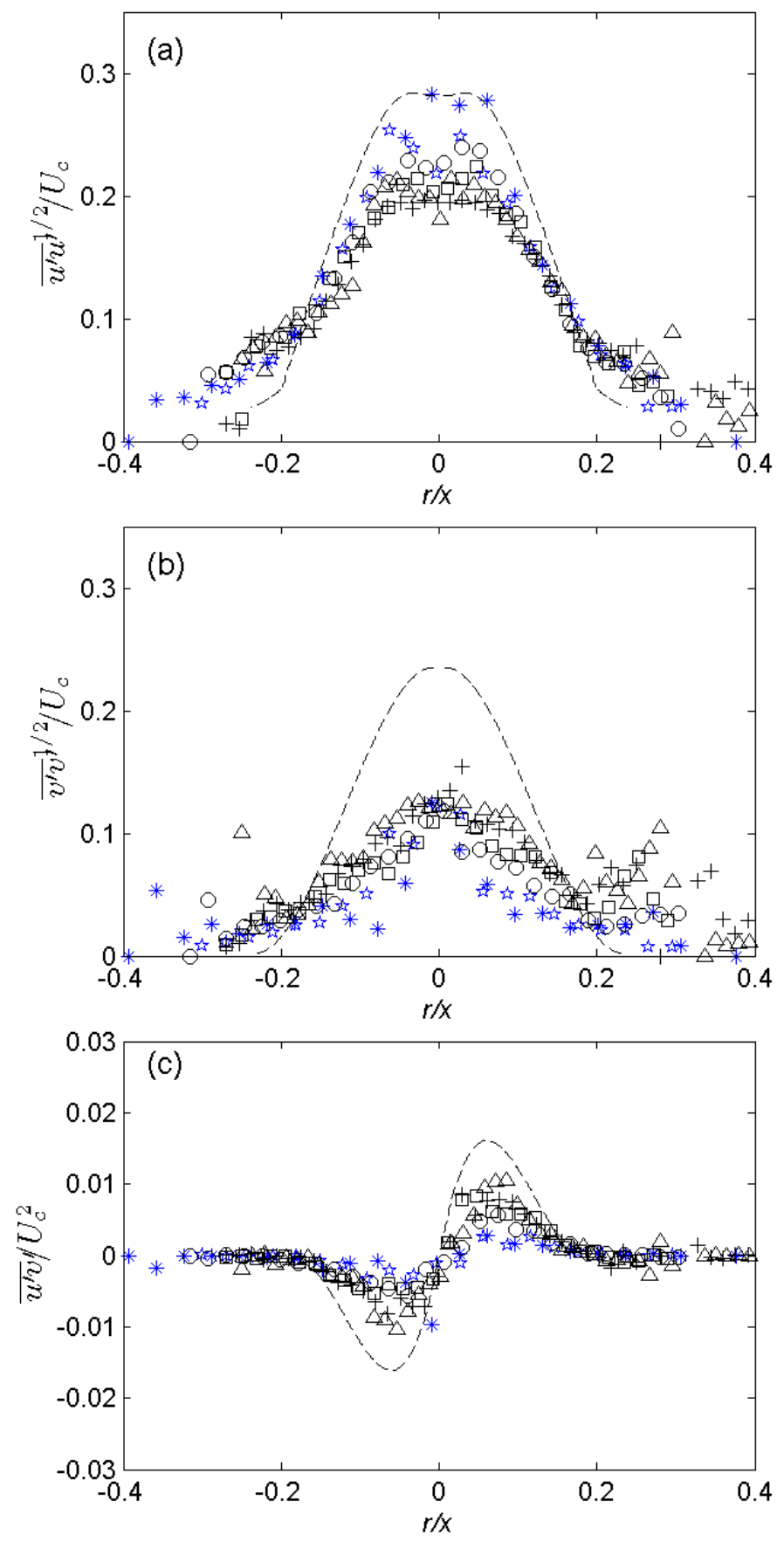

Fig. 6. Turbulent Reynolds stresses in a particle-free momentum jet. Symbols same as Fig. 5. Lines are data of Wygnanski and Fiedler (1969). 

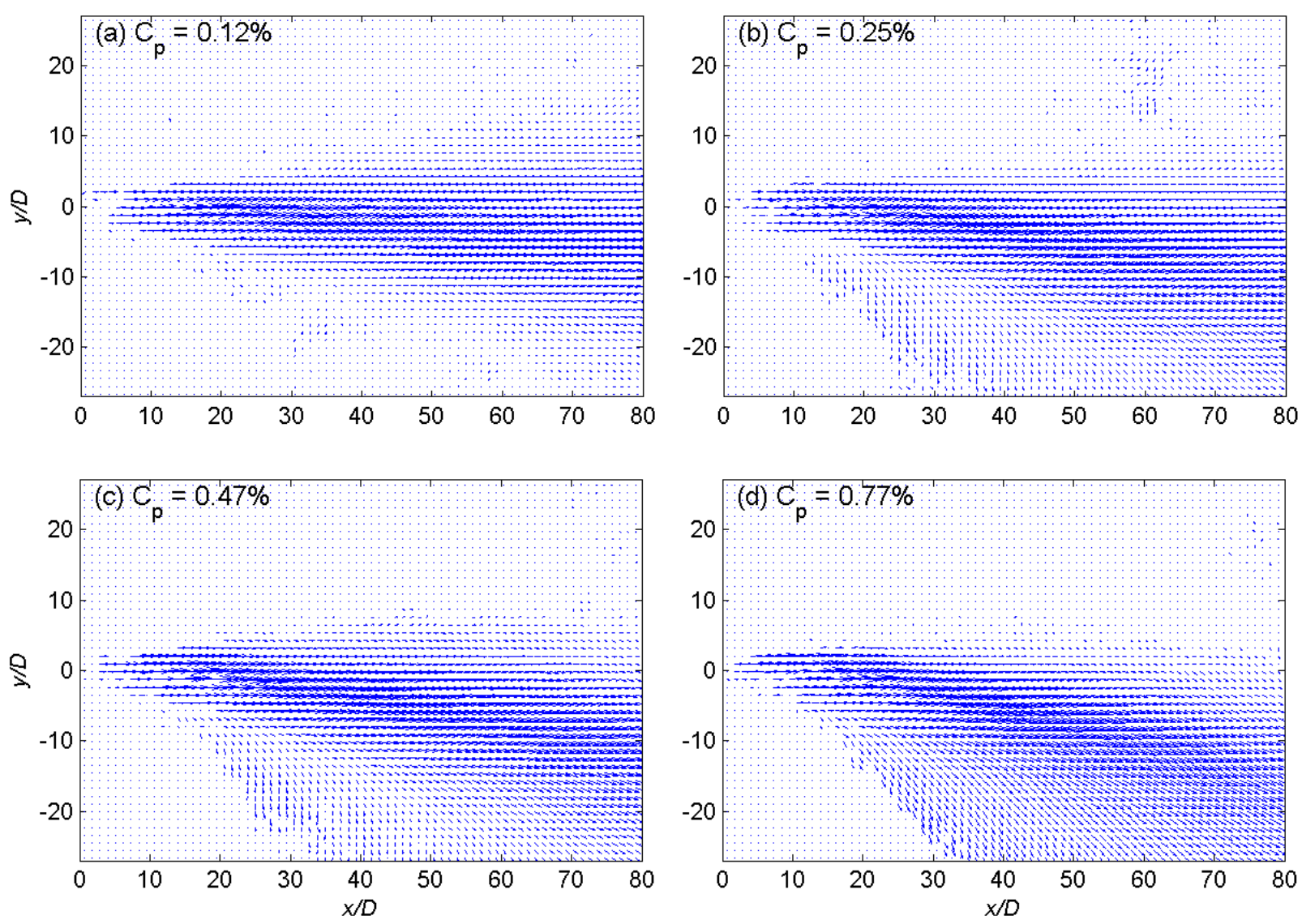

Fig. 7. Mean velocity field of jet fluid flow in sediment jets. 
(a) $C_{p}=0.12 \%$

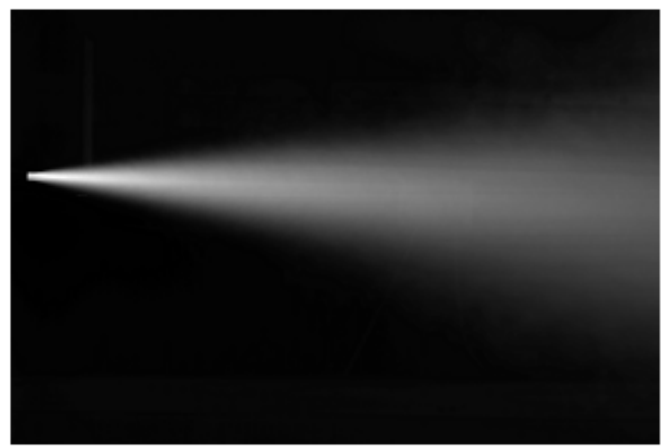

(c) $C_{p}=0.47 \%$

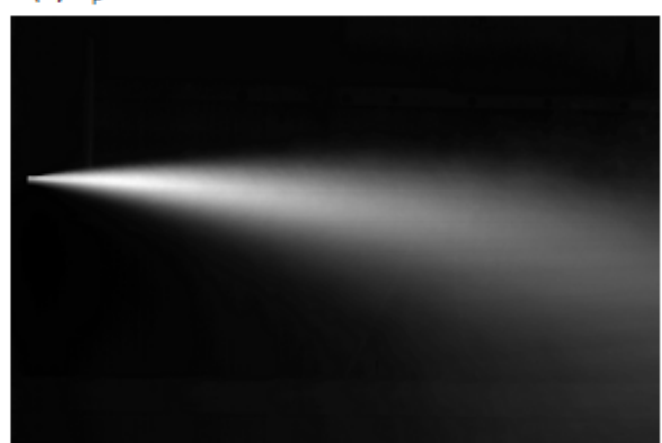

(b) $C_{p}=0.25 \%$

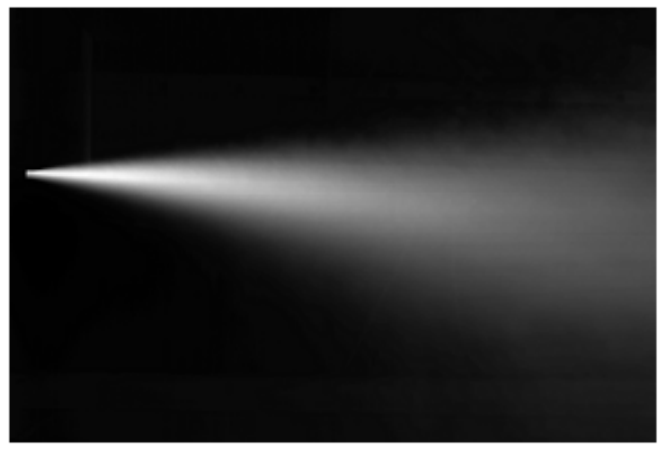

(d) $C_{p}=0.77 \%$

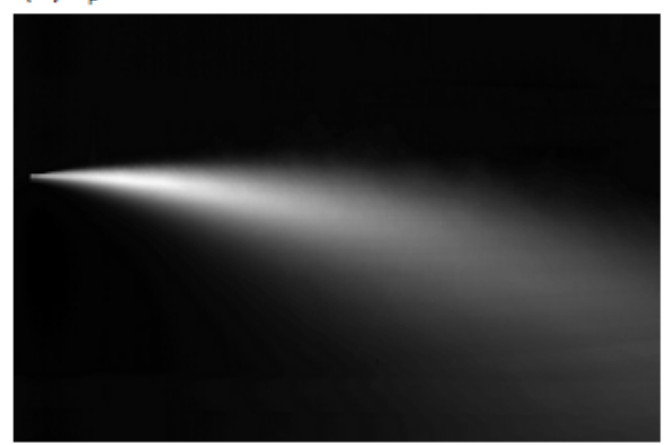

Fig. 8. Mean LIF visualization of jet fluid flow. 

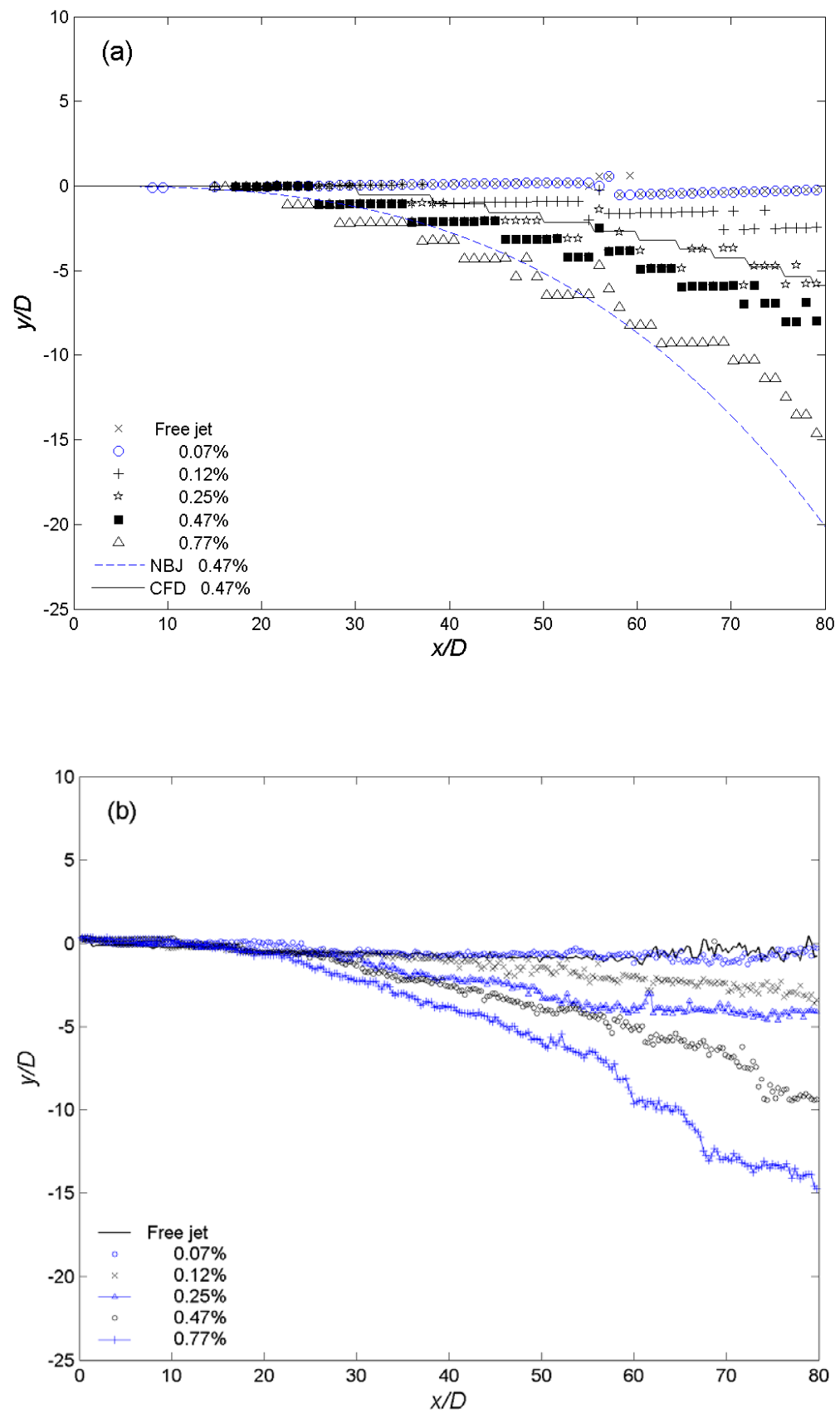

Fig. 9. Centerline trajectories of sediment jets. (a) from mean velocity field; (b) from mean LIF visualization. NBJ: JETLAG prediction of equivalent negatively buoyant single-phase jet; CFD: CFD result using drag forces between phases. 


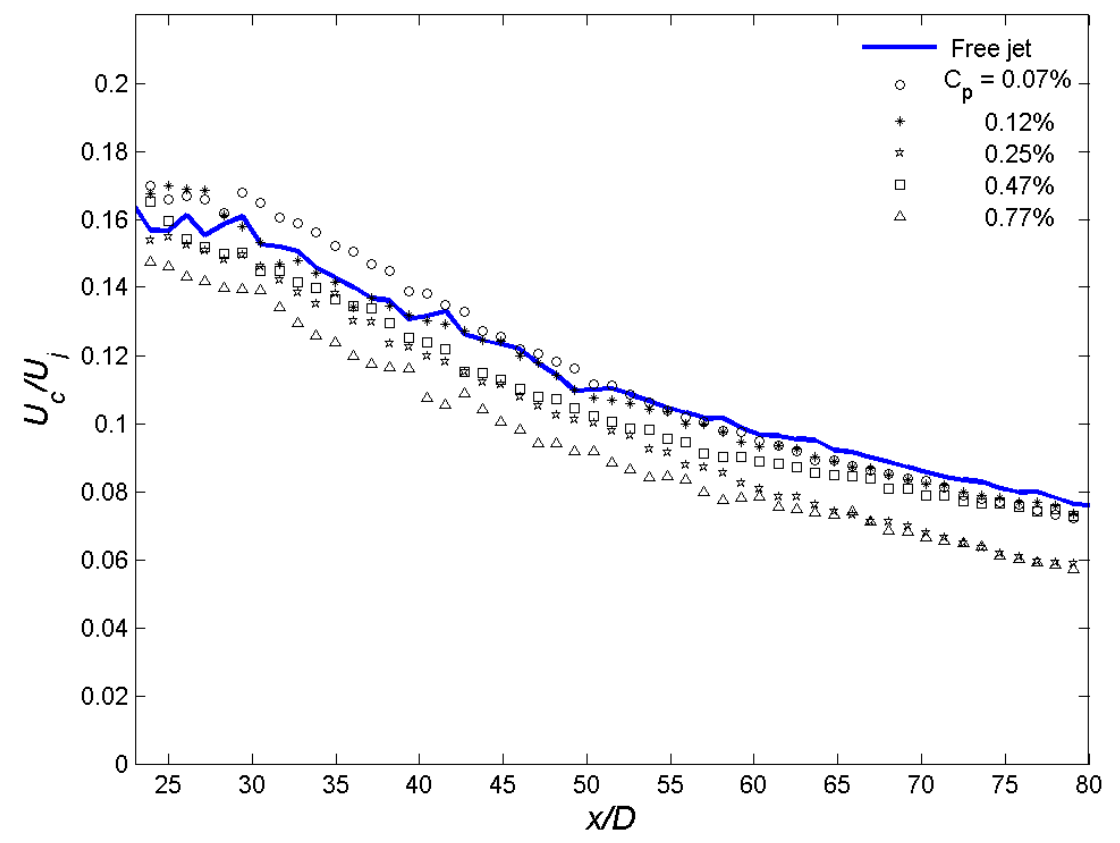

Fig. 10. Centerline velocity decay in sediment jets. 

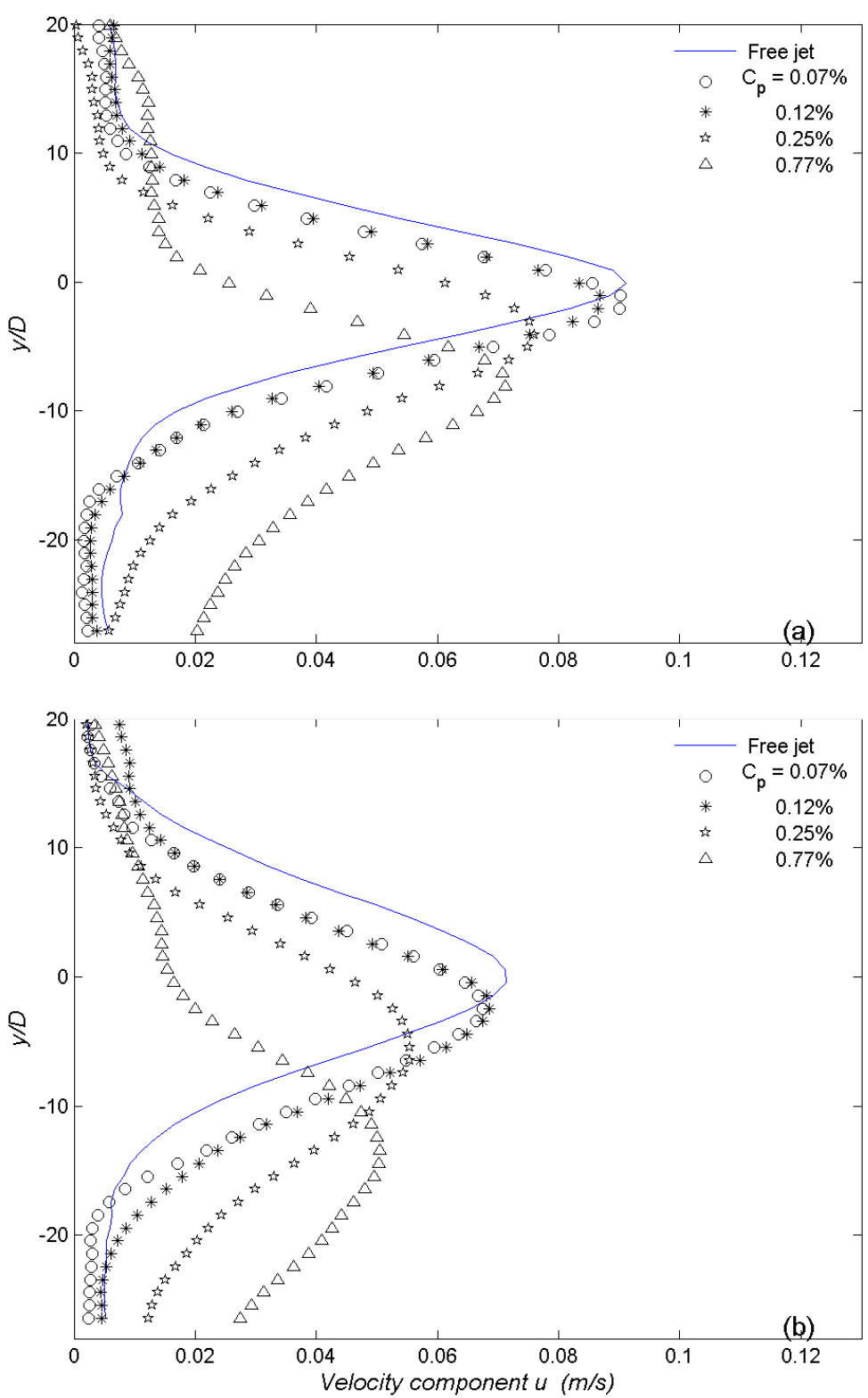

Fig. 11. Vertical profiles of mean axial fluid velocity. (a) $x / D=60$; (b) $x / D=80$. 

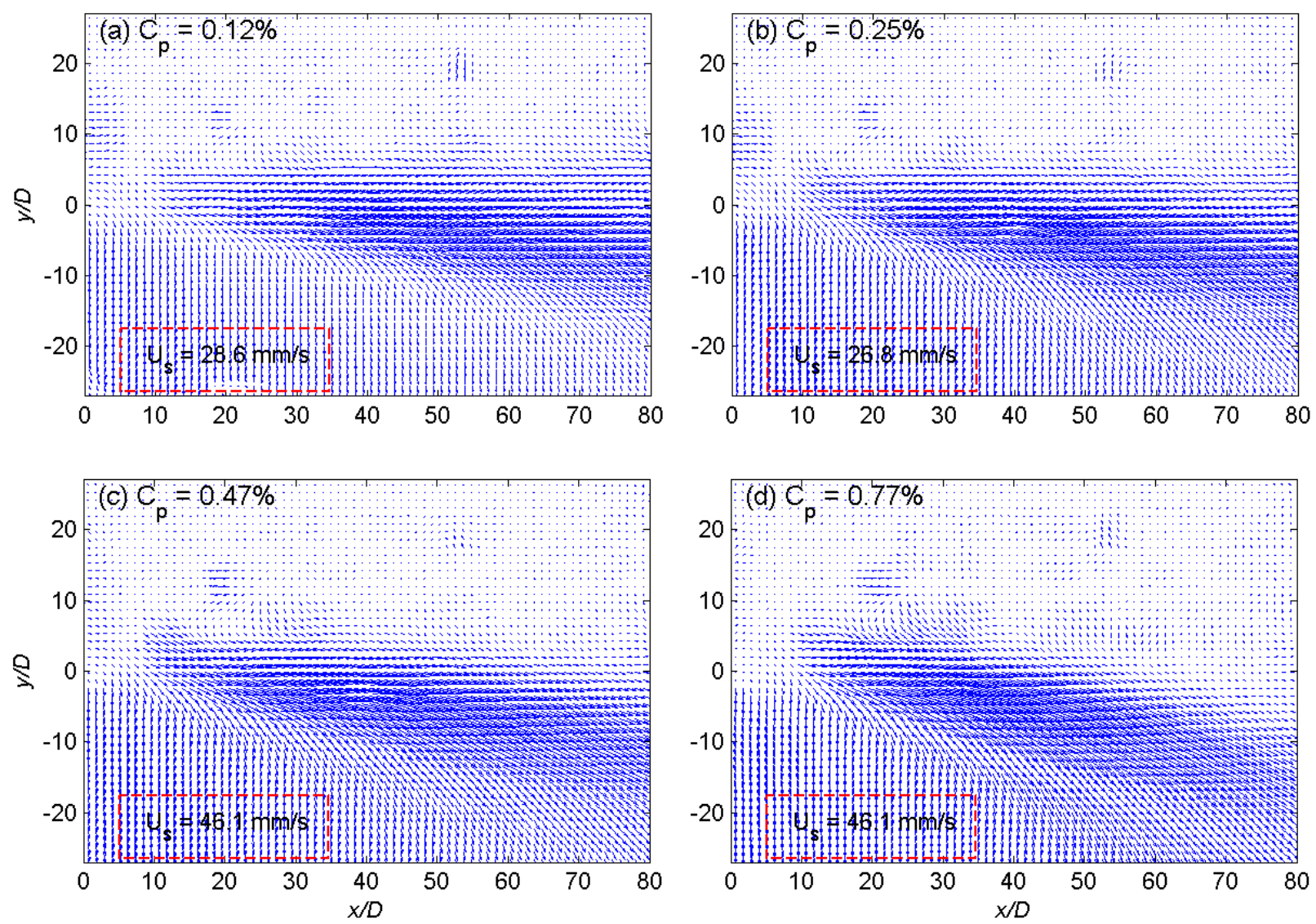

Fig. 12. Average velocity field of sediment particles. 


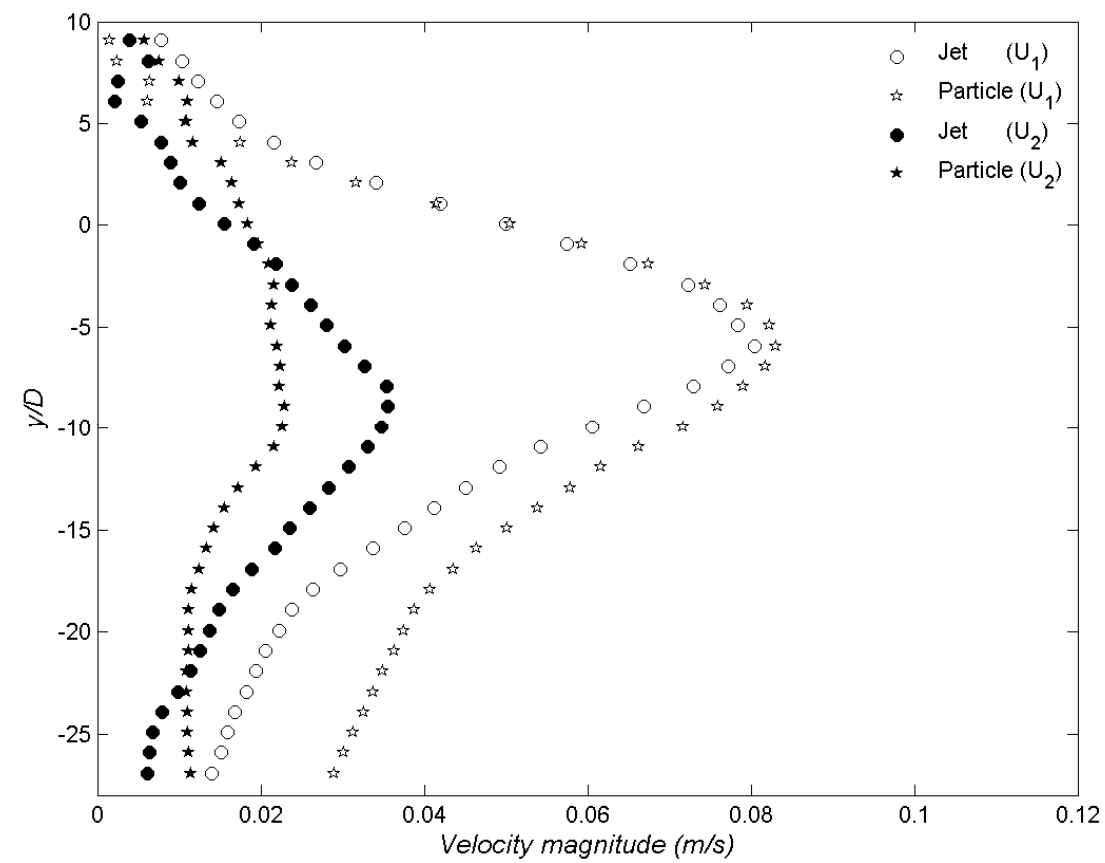

Fig. 13. Comparison between vertical profiles of mean fluid velocity and particle velocity at $x / D=65$. Open symbols: $U_{j}=U_{1}=0.95 \mathrm{~m} / \mathrm{s}$ and $C_{p}=0.47 \%$; solid symbols: $U_{j}=U_{2}=$ $0.45 \mathrm{~m} / \mathrm{s}$ and $C_{p}=0.52 \%$. 


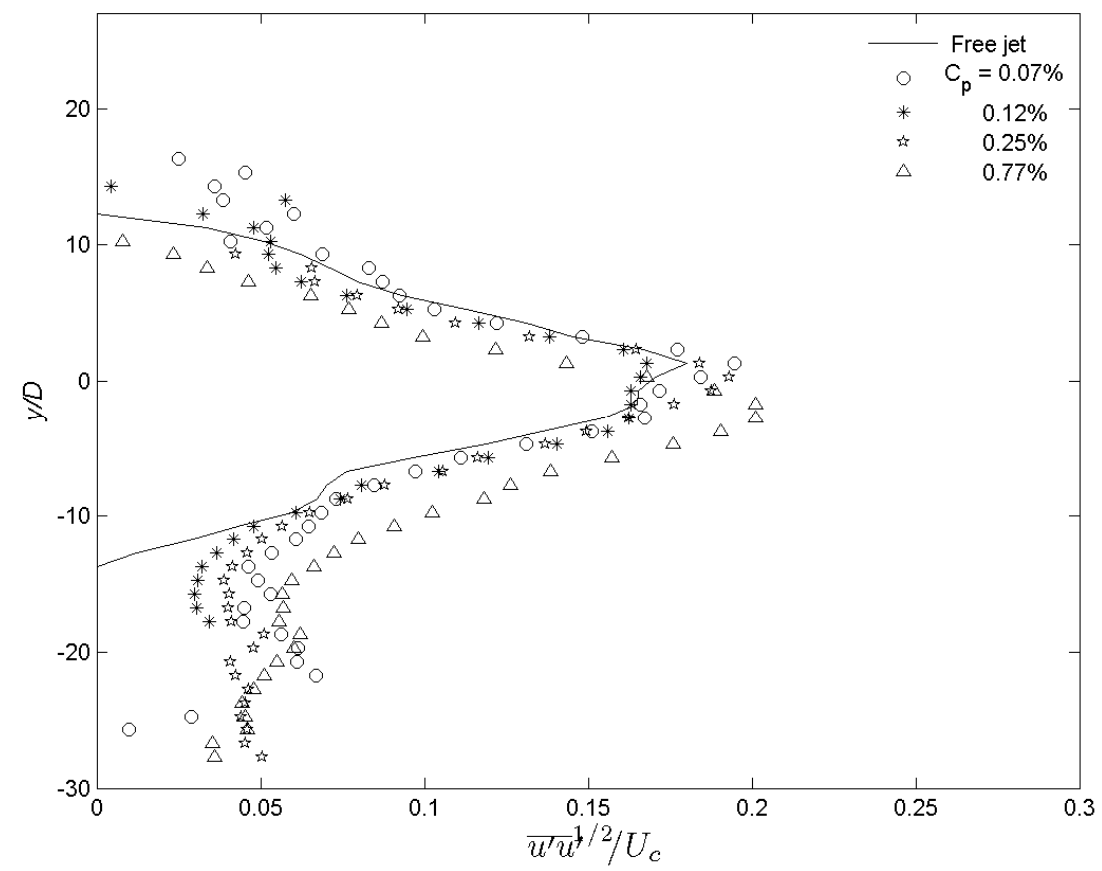

Fig. 14. Turbulence intensity affected by particle loading. $x / D=40$.. 

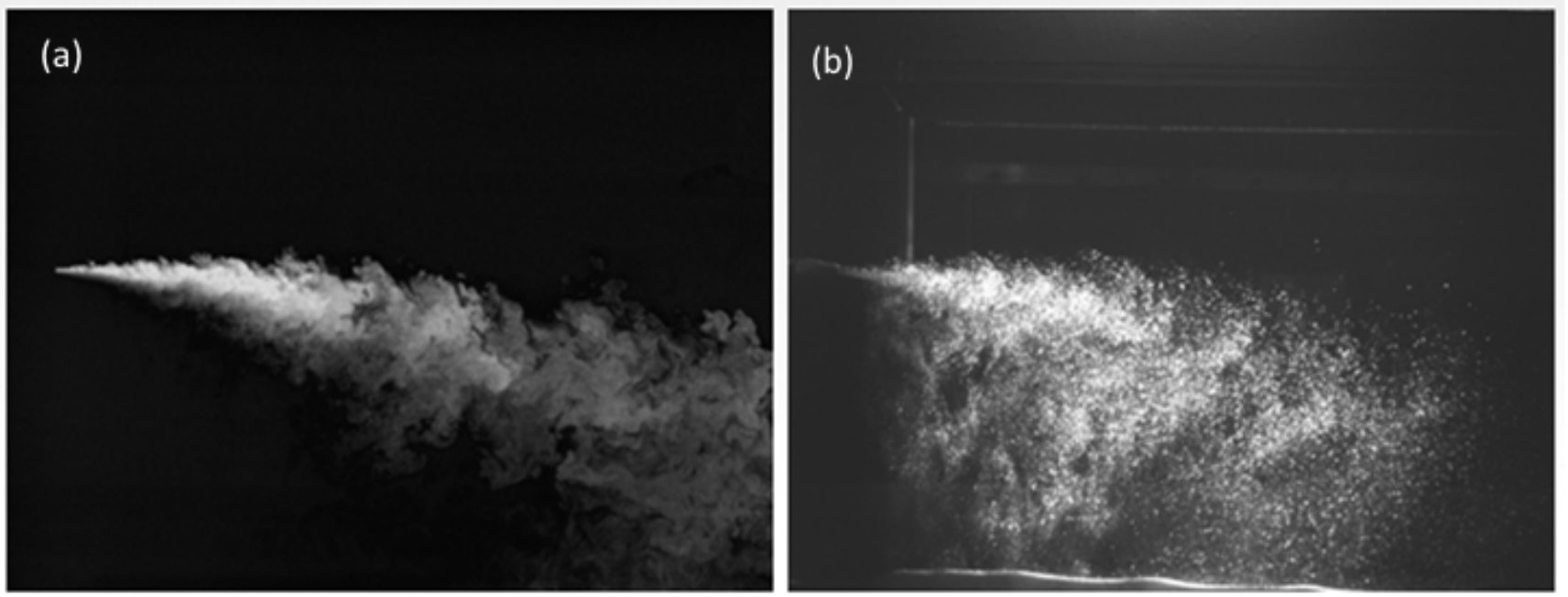

Fig. 15. Intense interaction shown from visualization. $C_{p}=0.77 \%$. 